\title{
The Belief of World Records Fusion and its Software to Cyber Protection
}

\author{
Geeta Sai Sruthi, A. Prakash, P. Padmaja, D.B.K. Kamesh
}

\begin{abstract}
Worldwide statistics Fusion is one of the foremost technologies utilized in complicated structures. While the software of information fusion has already proposed for the implementation of precise tools, its extension to the overall design system of a complicated system is some distance from a favored target. The improvement of advanced architectures based totally on an interdisciplinary design method makes this extension possible, in particular on the higher stages of the construction, related to scenario assessment, effect assessment, and method refinement. This paper analyses one of the superior cyber safety architectures and explores the functionality of this architecture to consist of records fusion gear at the top level of the design. The effects of the generalization of records fusion strategies are then analyzed, and the ensuing improvements in the network protection of vital infrastructures are described and quantified.

Keywords: Information fusion, cyber protection, situational attention, environment monitoring, essential infrastructures, community safety.
\end{abstract}

\section{INTRODUCTION}

Sensor subsystem, which collects facts coming from the external environment; b) Records and statistics Fusion subsystem, which merges statistics amassed through sensors and information and information arising from external intelligence resources; c) Human-Agent in the Loop, which plays operations with the aid of a human operator within the decision loop; d) Center Processor, which mixes all the facts produced through the preceding subsystems with internal points extracted through statistical studying, on the basis of the ancient database the above mention from(A to D) in an advanced security system for critical infrastructures shows in (Fig.1).

An interdisciplinary (human/gadget) method is used in the gadget, having to cope with the heterogeneity of the records produced with the aid of the machine itself, the ones collected through the tracking tools, and comfortable information coming from unique assets. Monitoring statistics derived from different forms of sensing devices, at the same time as

Revised Manuscript Received on December 22, 2019.

Geeta Sai Sruthi ${ }^{1}$, Department of CSE, Malla Reddy Engineering College for Women, Maisammaguda, Dhulapally, Kompally, Medchal (M), Secunderabad, Telangana 500100

A. Prakash ${ }^{2}$, Department of CSE, Malla Reddy Engineering College for Women, Maisammaguda, Dhulapally, Kompally, Medchal (M), Secunderabad, Telangana 500100

Dr. P. Padmaja ${ }^{3}$, Department of CSE, Malla Reddy Engineering College for Women, Maisammaguda, Dhulapally, Kompally, Medchal (M), Secunderabad, Telangana 500100

Dr. D.B.K. Kamesh ${ }^{4}$, Department of CSE, Malla Reddy Engineering College for Women, Maisammaguda, Dhulapally, Kompally, Medchal (M), Secunderabad, Telangana 500100 cozy statistics come from shrewd external assets, the human inside the loop dealers, and internal machine intelligence. Specific intelligence statistics and facts travel through the internet by analyzing messages exchanged through social networks. For the above motives, the utility of information Fusion to cyber safety systems for essential infrastructures wishes an international approach and its extension to the whole design manner.

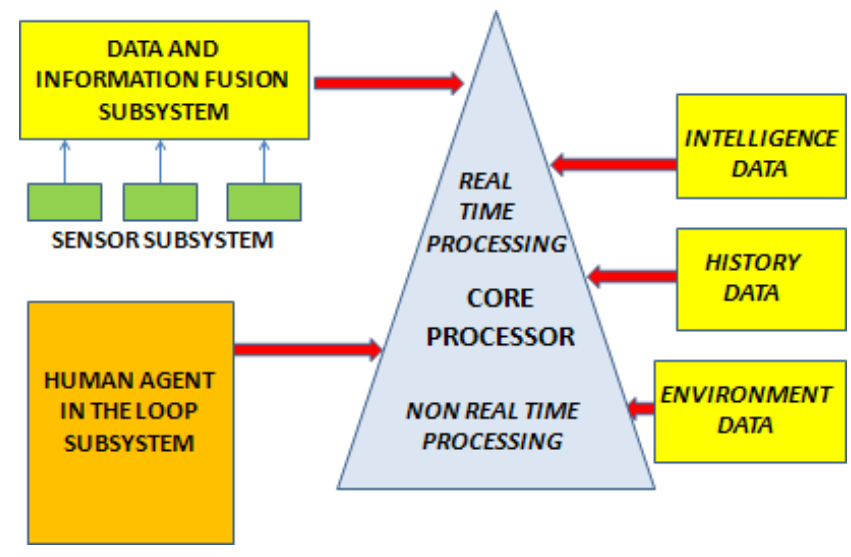

Fig. 1: Critical Infrastructures of Advanced Cyber Security Architecture

Giving the JDL definition of Data Fusion [7] (Fig. 2), Data Fusion is a multilevel process, with five different levels, ranging from Level 0 (lowest level) up to Level 4 (highest level).

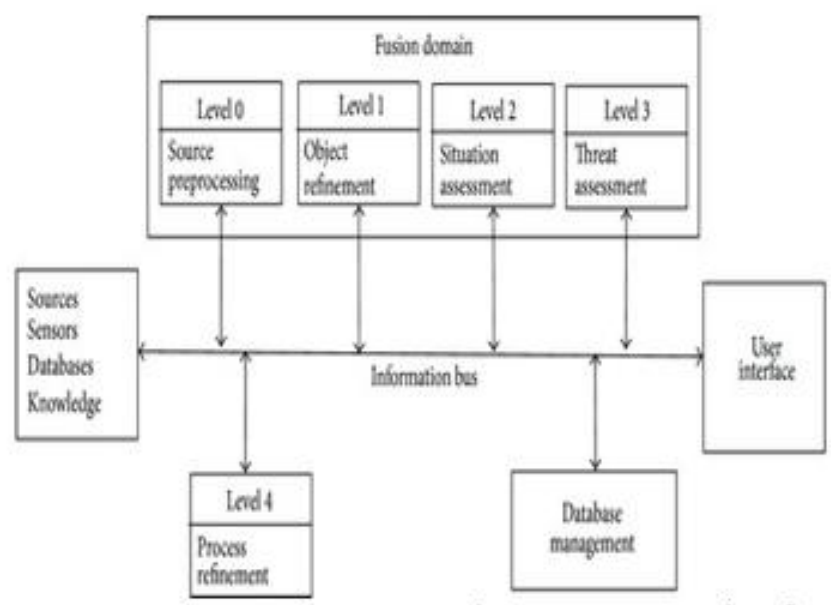

Fig. 2: The JDL Data Fusion Framework.

Level 0, source Preprocessing: consists of the facts extraction technique. This degree reduces the number of statistics and continues useful records for excessive-level tactics.

Level 1, item Refinement: employs the processed facts from the previous stage. 
The output outcomes of this level are the item discrimination (classification and identification) and item tracking (state of the object and orientation). This degree transforms the enter records into consistent data systems.

Level 2, in between the objects (i.e., proximity, verbal exchange), to determine the significance of the entities or gadgets in a selected environment. The purpose of this degree includes acting excessive stage inferences and figuring out sizeable sports and events (styles is preferred). The output is hard and fast of high-degree assumptions.

Level 3, impact evaluation: evaluates the effect of the detected activities to perform a future projection and become aware of possible risks, vulnerabilities, and operational opportunities. This stage consists of an assessment of the chance or danger and a prediction of the logical outcome.

Level 4, process Refinement: gives useful resource and senior management. The aim is to attain green aid control, even as accounting for challenge priorities, scheduling, and they manage resources.

The JDL version allows records of Fusion techniques to be extensively employed on multi-sensor environments to merge and aggregate facts from distinct sensors. Information Fusion in multi-sensor environments makes it possible to gain better detection possibilities and better reliability by way of the usage of records from more than one dispensed resources. The software of files Fusion techniques to the highest stages of the architecture (global facts Fusion) is the winning technique to design reliable and secure systems, by using overcoming the drawbacks nonetheless current in conventional cyber protection programs.

In this context, the next sections focusing the first review the application of Data Fusion to Lever 0 and 1(already implemented in the present state of the art systems), then we have to discuss on the implementation of Data Fusion Techniques to higher levels like Level 2 t0 4(Global Data Fusion concept) carried out the above construction.

The graphics reported in the rest of the paper are the result of merging and simplifying the data collected in a series of experiments on Global Data Fusion applied to critical infrastructures. The main scope of this discussion is to assess the improvement achievable in a cybersecurity system by implementing the Global Data Fusion idea

\section{LEVEL 0 AND 1: SOURCE PRE-PROCESSING AND OBJECT IMPROVEMENT}

The primary desires of the low JDL ranges (degree zero and level 1) are to become aware of, hit upon and symbolize the gadget surroundings, which includes cyber entities (e.g., computers, networks, information go with the flow, and so forth.), their relative statistics (e.g. working systems, hardware, patches, etc.) and intrusion detection records (e.g. security logs, adversary presence records, etc.). The device environment information might be a useful resource, both a cyber defense electronic structure or a human being able to manage a limited amount of detected attacks. To be more productive, Data Fusion has to be extended at higher levels, as explained in the next sections.

\section{LEVEL 2: SITUATION ASSESSMENT}

The level 2 manner combines the more than one function of a multi-sensor community right into a comprehensive picture of the cutting-edge scenario. Primarily, this method provides a shared knowledge of the cutting-edge kingdom of the machine. The gadget records, along with operating machine patches, hooked up antivirus software definitions, list of running techniques, and other safety-associated facts, supply an estimation of the device robustness and its ability to counteract a regarded set of attacks. The representation of these kinds of information for the entire network offers a unique estimation of the extent of consciousness inside the modern system nation.

In the famous case, machine focus is a mixture of two different factors, particularly device health evaluation and know-how of attacker competencies. The algorithms applied in stage 2 statistics Fusion are mainly sample matching and system learning. The assessment of the gadget fitness (e.g., patch level, antivirus definition, and so forth.) may be analyzed with recognize to an acknowledged preferred safety kingdom.

Suitable actions needed both when the desired country or if the machine reveals undesired behavior like high CPU load, low available pressure area, senior community visitors, and many others. As a result of those moves, the very last assessment acknowledges a suitable stage of the network protecting posture.

Moreover, there ought to be particular information on the abilities of the potential attacker, together with the estimation of the type of attacks that might be more likely to take place, by mastering from ancient information.

Stage two statistics fusion represents an increase past the advent of uncooked sensor records, as happens at the primary level, and helps the synthesis of more much vital information for directing human selection-making. Bayesian decision idea is one of the most common strategies employed in level two statistics fusion. It's miles used to generate a probabilistic version of unsure machine states by way of consolidating and deciphering overlapping records provided through several sensors. It additionally determines conditional probabilities from a priori proof. This stage is used one among famous maximum strategies, which are: Bayesian decision concept and Dempster-Shafer Evidential Reasoning.

The Bayesian Networks Bayesian networks are beneficial for both inferential explorations of previously undetermined relationships among variables as well as descriptions of those relationships upon discovery. The Dempster-Shafer method has numerous advantages over the Bayesian choice concept. Most importantly, hypotheses do now not have to be collectively distinct, and the probabilities concerned can be either empirical or subjective. As Dempster- Shafer sensor detailed information at various tiers of abstraction, a priori expertise may be accessible.

\section{SYSTEM AWARENESS}

The mixture of the native country of the protecting posture and the capability set of the capability attackers effects inside the definition of the attention of the current device protection level. Gadget attention entails three critical regions, namely computing and community additives, chance statistics, and venture dependencies. 
Accomplishing a high stage of device recognition calls for consciousness on statistics collection, statistics control, and surroundings analysis to get an actual-time photograph of the situation, mainly about pc systems, networks, and customers. Device attention includes three sub-subjects, specifically community cognizance, risk awareness, and project consciousness. Network cognizance: disciplined asset and configuration control, recurring vulnerability auditing, patch control and compliance reporting, apprehend and share incident attention across the enterprise. Chance attention: discover and track internal incidents and suspicious behavior, include knowledge of outside threats, participate in go-industry or move-authorities chance- sharing groups on viable indicators and warnings. Mission focus: develop a comprehensive image of the critical dependencies to function in cyberspace, understand these vital dependencies to guide task impact in forensic analysis (after a state of affairs); triage and real-time disaster motion response (throughout a scenario); chance readiness evaluation prior to project execution (awaiting and heading off situations) and knowledgeable protection planning (getting ready to mitigate the effect of a future position).

A standard pattern of the system attention as a function of the variety of environment parameters beneath manipulate pronounced in discern 3 , in which the machine attention degrees from zero (no care) to at least one (whole focus) and the parameters of the surrounding consist of both machine fitness parameters and external risk parameters.

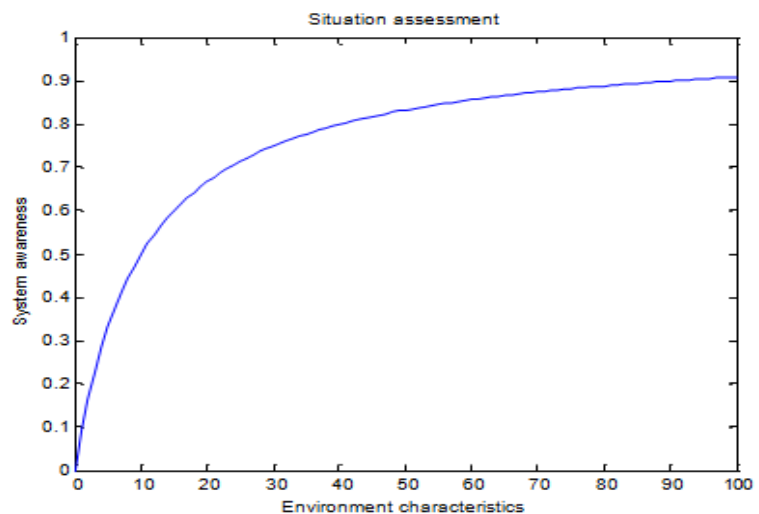

Fig. 3: Environment Characteristics of System awareness as a function

\section{LEVEL 3: IMPACT ASSESSMENT}

The level three-technique has the aim to coordinate the defensive movement of the community after an appropriate assessment of the feasible attacker's options. This task is predicated at the gadget attention gained from stage 2 and exploits an also expertise about common vulnerabilities and exposures of the network, together with a deep understanding of the attacker's strategies. An automatic fusion technique combines the knowledge about the abilities of the assault deriving from previously learned techniques with the estimation of the present-day health of the community additives. As mentioned in the addition step, the safety device, with the possible intervention of the human analyst (through a human within the loop sub-method), presents additional moves to recover the device and neutralize the effects of the assault. With a purpose to carry out the protection motion, it is paramount to apprehend as plenty as possible the feasible attacker's techniques and the multiplicity of protection abilities of the system. The level 3 manner extends the present day scenario into the future to draw inferences about threats and possibilities for operations. The most used techniques on this stage are professional systems, Blackboard structure, and Fuzzy good judgment. A professional gadget as the personification inside a pc of expertise primarily based factor from a professional skill in the sort of form that the machine can offer shrewd advice or take an intelligent choice approximately processing feature. A blackboard system includes 3 most important components: the software expert modules, presenting particular know-how wished by way of the application, the blackboard, specifically a shared repository of problems, partial solutions, suggestions, and contributed data and the manage shell, which controls the go with the flow of trouble fixing hobby inside the gadget. Fuzzy good judgment is a mathematical technique for coping with imprecise records and problems which have many solutions in preference to one.

\section{PROBABILITY OF RECOVERY}

The impact assessment/ threat refinement analysis contains a variety of threat perspective models, to derive associate degree estimation concerning the likelihood of recovery, just in case of attack. These models type a central repository of threat intent illation info. Every model \{is concerned cares thinks concerning worries is bothered \} with reasoning about the adversary's strategy from one analysis perspective. A ranked graph structure delineates every threat perspective model. The graphs give a structure for aggregation fact lets below a particular view and so reasoning on the probability of 1 or additional soul strategy. The machine mechanisms to perform the fusion of fact let proof inside every graph square measure provided.

A typical situation, expressed in terms of probability of recovery, as a function of the threat capabilities and the multiplicity of the network defense resources, is represented in Figure 4, where the likelihood of recovery, ranging from 0 (no improvement) to 1 (full recovery), is a function of both the threat capability and the multiplicity of the network defense capabilities. In Figure 4, where the probability of recovery, ranging from 0 (no improvement) to 1 (full recovery), is a function of both the threat capability and the multiplicity of the network defense capabilities.

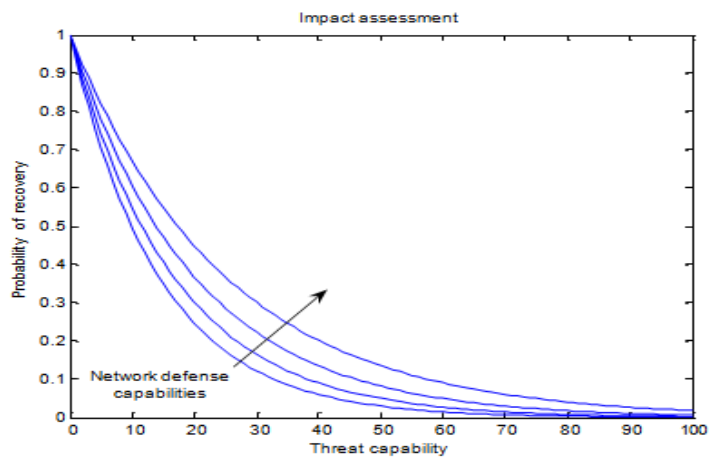

Fig. 4: Probability of restoration as a function of threat capability and network defense capabilities. 


\section{LEVEL 4: PROCESS REFINEMENT}

The standard four systems is specifically a selection system, concerning the statement of the overall facts fusion gadget and the selection of the abilities to discover new assault techniques. This decision technique consists of tracking for precise types of traffic within the community, inclusive of connections to unknown hosts or servers in foreign countries, or an excessive wide variety of contexts through the parallel ports, and many others. Attributable to this investigating hobby, which also can be performed in a human within the loop mode, the extent four methods can deploy particular tracking gear to make the same system extra green. According to corridor \& McMullen (2004), human pc interaction (HCI) research within the fusion area has primarily considered the interaction between the consumer and a geographical records display (based totally on the machine of a geological record) thru menus and dialogs. But, the modern-day studies hobby in this region is developing, and strategies which include gesture recognition and herbal language interplay are currently of a hobby.

\section{PROBABILITY OF BLOCKING THREATS}

Process refinement can then be defined as a meta-process or as a decision making the task, taking viewpoint from decision theory, determining the most appropriate sensor action to be taken to achieve maximum utility. The application of this level to the whole Data Fusion process serves to increase. The application of this level to the total information Fusion method serves to extend the likelihood of interference threats, by knowing the potential threat choices and customizing the active defense tools to deploy the first practical observation tools. A general pattern of the likelihood of interference threats as a operate of the number of choices in hand by the risks in restraint is according in Figure five, wherever the danger defeating likelihood, ranges from zero to one, maybe a operate of each the multiplicity of threat choices and also the multiplicity of observation tools deployed by the system.

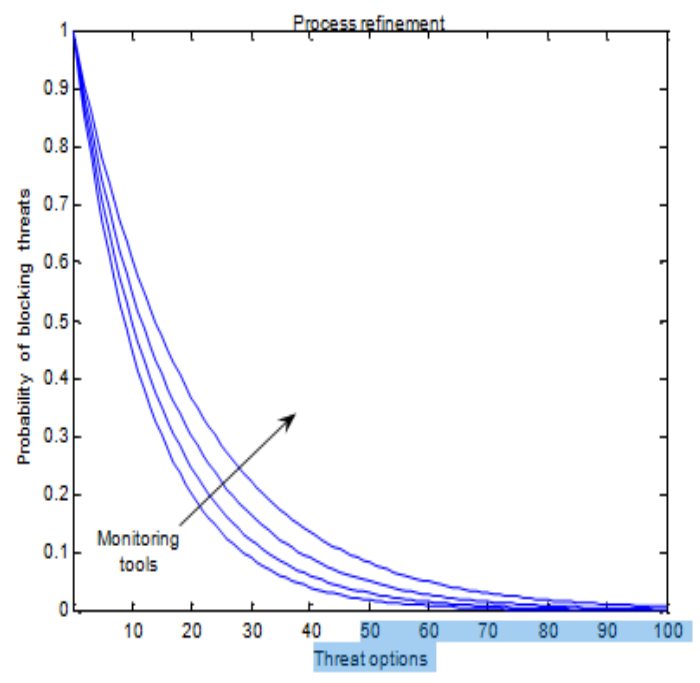

Fig. 5: Probability of blocking threats as a function of threat options and monitoring tools.

\section{PERFORMANCE EVAlUATION}

Within the last sections, we've got explored the functionality of a multi-sensor structure based on an interdisciplinary design technique to take advantage of the worldwide information Fusion concept. The consequences of this new kind of records fusion techniques were analyzed little by little, if you want to acquire some measure of the development inside the network safety of critical infrastructures, resulting from the software of those techniques. This phase has the goal to refine this estimation, counting on the previous findings of system attention, the possibility of recovery and the chance of blocking off threats.

So one can attain the very last results. We will, in short, resume the achievements contained within the previous four sections (phase 2, segment 3, phase four and chapter five).

In section 2, we reviewed the responsibilities completed at the bottom information Fusion stages. In chapter three, we showed that the device focus grows up with the multiplicity of surroundings parameters underneath manage. Machine attention includes each information of the risk abilities and expertise and lead of defense skills. As a lot as the network is aware of hazard abilities, as more effectively, the community can installation its protection skills. In phase 4 , we showed that while controlling both hazard capability and protection functionality, the network can reach a high stage of the possibility of restoration from external attacks. Fast recovery from outside attacks corresponds to leave the attacker with much fewer alternatives available. In Section 5, we discussed the network capacity of blocking the maximum number of threats that can attack the system. Leaving the danger with fewer options and using as much as possible suitable monitoring tools contribute to achieving more efficiency in blocking threats and, as a consequence, to reach a higher level of security. After merging the results of Section 2, Section 3, Section 4, and Section 5, we produce a final estimation, which is graphically represented in Fig. 6. In particular, we evaluate the increased network security as a function of the data fusion capabilities of the network and the number of threats that can potentially attack the system.

The final estimation shows that the network security can increase sensibly as much as the system can rely on a high number and quality of data fusion capabilities and, as much as the system can exploit a high level of threat detection capability.

\section{INCREASED NETWORK SECURITY}

The effect of the application of the highest levels of the Data Fusion techniques has been analyzed step by step, to find the improvements in the network security of critical infrastructures as a consequence of this improved process. A general pattern of the increased network security as a function of the Data Fusion capabilities and the threat detection capability is reported in Figure 6. 


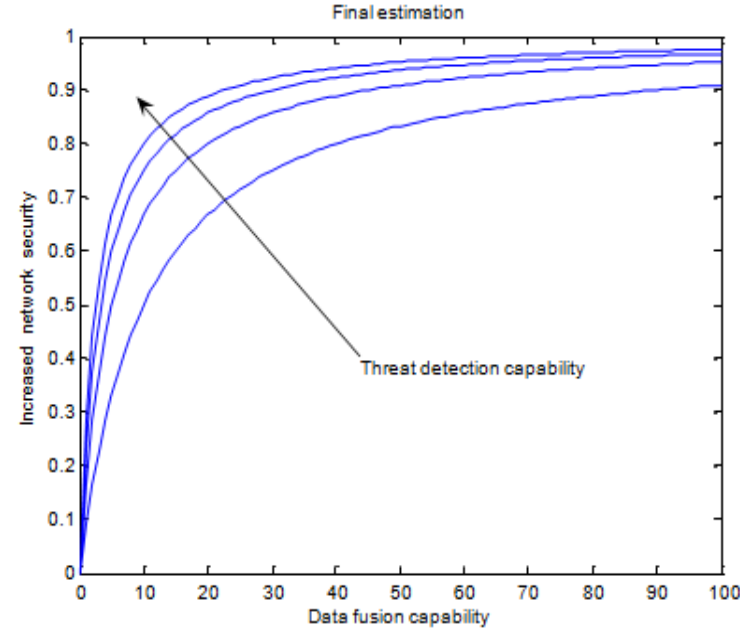

Fig. 6: Increased network security as a function of data fusion capabilities and threat detection capability

We observe that, in a very general case, wherever the information fusion techniques delineated during this paper don't seem to be applied, the network security is calculable as having performance figures happiness to the left bottom section of the graphic in Figure half-dozen. Once using our fusion ways, network security grows up and reaches the highest sector of identical graphics. An extra thought that was already introduced within the last sections regards the cooperation between human and machine, that is important to realize the measurable results. Although the human contribution cannot be embedded in our measurements, the collaboration between human and machine is decisive to understand the most level of security.

\section{CONCLUSION}

The implementation of the overall variety of facts fusion capabilities for cyber safety continues to be an open difficulty. Information Fusion algorithms want to be developed in any respect ranges of the JDL model. The latest development in advanced cyber safety architectures, which makes use of a unique interdisciplinary technique, allows extending information Fusion to the higher degrees of the JDL structure, particularly scenario evaluation, impact assessment, and process refinement. This paper specializes in a proposed cyber safety structure and explores the effectiveness of this extension on the top degree of the structure. Concerning situation assessment (Level 2 in the JDL model), a general pattern of the system awareness as a function of the number of environment parameters under control is derived, where system awareness is found as substantial dependant from the accurate knowledge of the environment characteristics.

Impact assessment (Level 3 in the JDL model) corresponds to the Data Fusion capability, which coordinates the defensive action of the network utilizing the knowledge of the attacker techniques. Our analysis shows that the probability of recovery can be sharply increased by a suitable number of defense measures and consistently decreased by threat capabilities.

Regarding process refinement (Level 4 in the JDL model), the goal is to analyze how much the probability of blocking threats can depend on the threat capabilities and defense capabilities.

At the end of the overall analysis, we show that the network security can be consistently increased by applying more data fusion capabilities and by incrementing the network capability of threat detection through a suitable combined process based on the Global Data Fusion concept.

\section{REFERENCES}

1. M. LaManna "Urban Environment Monitoring: System and Technology Issues," IMCIC 2012, 25-28 March 2012, Orlando, FL.

2. M. LaManna "Data Fusion of Heterogeneous Sensors in Urban Environment Monitoring" IMCIC 2013, 9-12 July 2013, Orlando, FL.

3. M. LaManna "Cost-Benefit Analysis of Automated Systems for the Control of Urban Critical Infrastructures” WMSCI 2015, 12-15 July 2015, Orlando, FL

4. M. LaManna "Future Trends for Cyber Security for Critical Infrastructures" WMSCI 2016, 5-8 July 2016, Orlando, FL.

5. M. LaManna "Technology Intercepts for Cyber Security applied to Critical Infrastructures” WMSCI 2017, 8-11 July 2017, Orlando, FL.

6. JDL, Data Fusion Lexicon. Technical Panel For C3, F.E. White, San Diego, Calif, USA, Code $4^{20}, 1991$.

7. S. Schreiber-Ehle, W. Koch "The JDL model of data fusion applied to cyber-defense - A review paper," Workshop

8. on Sensor Data Fusion: Trends, Solutions, Applications, Bonn, 4-6 September 2012

9. T. Amending: "Cybercrime: much more organized," CSO Report, 23 June 2015.

10. I. Hashem et al. "The rise of Big Data on Cloud Computing: Review and open research issues," Information Systems, Vol. 47, January 2015.

11. B. Berkowitz "Intelligence for the Homeland." SAIS Review of International Affairs 24, no. 1, 2004.

12. W.Karwowski "International encyclopedia of ergonomics and human factors," CRC Press, 2006.

13. Kalyan Veeramachaneni, CSAIL, MIT, et al. "AI2: Training a big data machine to defend." AI2 IEEE International Conference on Big Data Security, April 2016, New York.

14. I. Arel, D. C. Rose, and Thomas P. Karnowski "Deep Machine Learning. A New Frontier in Artificial Intelligence Research" IEEE Computational Intelligence Magazine, 2013.

15. G. Dahl, W. Stokes, Li Deng, Dong Yu, "Large-Scale Malware Classification using Random Projections and Neural Networks," IEEE Conference on Acoustics, Speech, and Signal Processing, 2013.

16. E. Blasch et al. "High-Level Information Fusion Management and System Design.” Norwood, MA: Artech House Publishers, 2012.

17. P. Shinkman: "Reported Russian Cyber Attack Shuts Down Pentagon Network," US News, 6 August 2015.

18. E. David, "Deep Learning for Automatic Malware Signature Generation and Classification," IEEE Intl. Conference on Neural Networks, Killarney, Ireland, July 2015.

19. Andler, S. F. Information Fusion from Databases, Sensors, and Simulations, Annual Report 2005, June 2006

20. Hall, D. \& Llinas, J. Handbook of multisensor data fusion. CRC Press.

21. Hughes, T.J. "Sensor Fusion in a Military Avionics Environment." Measurement and Control. Sept. 1989.

22. Hall, D. \& McMullen, S.A.H. (2004) Mathematical techniques in multisensor data fusion. Artech House.

23. Hughes, T.J. "Sensor Fusion in a Military Avionics Environment." Measurement and Control. Sept. 1989

24. Ramsvik, H. AIS as a tool for Safety of Navigation and Security Improvement or not?

25. Svensson, P. Technical survey and forecast for information fusion. In RTO IST. Symposium on Military Data and Information Fusion. 20-22 October 2003.

26. Wald L., 1999, Some Terms of Reference in Data Fusion, IEEE Transactions on Geoscience and Remote Sensing Vol.37 No.3 May 1999. 